\title{
La crise des valeurs : l'éducation désacralisée dans L'homme qui m'offrait le ciel de Calixthe Beyala
}

\author{
Flora Amabiamina \\ Université de Douala, Cameroun
}

D

epuis la seconde moitié du $\mathrm{XX}^{\mathrm{e}}$ siècle, l'éducation, plus particulièrement celle des enfants, est devenue une préoccupation majeure de la société comme le montre Hannah Arendt dans un essai, La crise de la culture'. De fait, l'avènement du postmodernisme et le monde nouveau auquel il oblige les individus, du fait des bouleversements qu'il a entraînés, se ressent fortement dans l'éducation des plus jeunes. Ces mutations retiennent de plus en plus l'attention de pédagogues, sociologues et autres observateurs des liens sociaux.

Lorsqu'on parcourt un certain nombre de travaux effectués par les spécialistes, notamment des sciences de l'éducation, un constat se dégage : la perte progressive du pouvoir disciplinaire que l'ancien ordre social avait conféré aux parents, une sorte de dévaluation de l'autorité parentale. En effet, la marchandisation de l'ordre social qui ne va pas en ralentissant, du fait de la quête de plus en plus prononcée de conditions de vie meilleures par les individus, n'est pas sans modifier les rapports interpersonnels. Il en est ainsi dans le cadre familial où les parents, soucieux d'assurer le bien être matériel de leurs enfants, sont appelés à travailler davantage et, en conséquence, à passer plus de temps en dehors de leur foyer. Ils manquent, par la même occasion, les chances d'assumer décemment et avec assurance l'éducation de leur progéniture. Cette dernière, souvent abandonnée à ellemême acquiert une autonomie, ainsi gracieusement offerte, qu'elle n'entend céder à quiconque (que ce soit le parent qui veut reprendre à bon droit sa place ou l'institution scolaire qui participe à la suite des parents à l'éducation) lorsqu'elle en vient être menacée.

1 Hannah Arendt, La crise de la culture, Paris, "Folio ", 1972. 
L'homme qui moffrait le ciel nous édifie sur cette lente dégradation de l'institution familiale occasionnée par le postmodernisme. La famille constitue la base de la construction identitaire, en ce sens qu'elle offre à l'individu des valeurs qui lui servent de repères. Dans la relation mère-fille campée par l'héroïne Andela et sa fille Lou, l'on peut prendre la mesure des coups portés par le postmodernisme, et les valeurs que ce courant véhicule, à l'éducation. Et c'est la narratrice qui, dans un moment de désarroi, le donne à sentir lorsqu'elle en arrive à maudire le ciel de lui avoir donné son indomptable fille. Elle s'inscrit alors, comme elle-même le relève, parmi ces nombreux parents qui endurent les mêmes désagréments infligés par leur malicieuse progéniture. C'est pourquoi elle songe sérieusement à l'envoyer en Afrique pour y subir une séance de désenvoûtement. Elle semble arguer alors que Lou a besoin d'être confiée aux bons soins d'un exorciste. Mais il faut y voir, d'une part, une métaphorisation et une euphémisation de la nécessité de la prise en charge véritable de cette enfant terrible ce que, manifestement, elle admet dans son for intérieur mais se refuse à confesser ouvertement au lecteur. D'autre part, il faut lire implicitement dans le message véhiculé derrière cette attitude de la narratrice, sa reconnaissance d'une différence dans les modes d'éducation suivant les espaces en l'occurrence, pour ce qui nous concerne dans le cadre de cette analyse, l'Europe et l'Afrique. Même si elle ne le décline pas explicitement, le sousentendu peut signifier que la préférence ${ }^{2}$ de l'héroïne va à l'éducation africaine réputée pour être moins permissive.

La question centrale que nous entendons explorer ici est celle de savoir si le rôle des parents dans l'éducation ne se réduit-t-il (ou ne se réduira$\mathrm{t}$-il) pas désormais à la satisfaction des besoins matériels de leurs progénitures aux dépens de leur bien être psychologique, mental ou moral? Nous verrons ainsi que l'affaiblissement du lien entre les parents et les enfants est largement tributaire de l'espace social et de l'évolution des temps avec tout ce que cela induit notamment dans le domaine des valeurs sociales, particulièrement les valeurs éthiques. Et comme l'ont constaté

2 Même si on peut douter sérieusement de ce que la narratrice songe véritablement à éduquer sa fille à l'africaine du moment qu'elle n'a du continent noir qu'une vision caricaturale et exotique qui ne s'éloigne pas de la littérature afropessimiste et tropicaliste dont l'écrivaine est l'une des figures emblématiques sur la scène parisienne avec les clichés véhiculés tels l'Afrique de la pauvreté et de la misère, l'Afrique des sorciers et des marabouts, la Mecque de la dictature, le Goulag du féminisme, la Bastille de l'oppression des femmes et des enfants, le Vatican de la phallocratie et du machisme... 
certains sociologues et psychologues, la démission des parents de leur rôle en tant que premiers éducateurs de leurs enfants accentue cette crise de l'éducation.

\section{Espace social et crise de l'éducation}

Les penseurs sociaux s'accordent sur le fait que l'espace social et les temps, par les valeurs qu'ils véhiculent dans des périodes, participent mais influencent aussi, notablement, les façons d'être des individus. Les enfants, et particulièrement les adolescents, en plein processus de formation et pour cela en quête d'ancrage et de repères, représentent la population la plus vulnérable et la plus influençable. Les valeurs humaines sont tenues généralement comme participant des fondements des univers sociaux, même si elles ne demeurent pas statiques du fait qu'elles évoluent au gré des temps. S'agissant de L'homme qui m'offrait le ciel, si la narratrice se situe à cheval entre la culture africaine dont elle est originaire et la culture occidentale (française) qu'elle a adoptée, sa fille, en revanche, ne semble n'avoir été éduquée qu'à la lumière des valeurs sociales et culturelles françaises dont le pilier est la liberté. En effet, l'exemple de Lou contredit incontestablement les observations de sociologues français à l'instar de Bourdieu et Passeron ${ }^{3}$, Durkheim ${ }^{4}$ ou encore Foucault ${ }^{5}$ qui ont posé comme principe fondamental de l'éducation la discipline et la punition des enfants qui sont alors loin d'être des sujets libres et indépendants du pouvoir des parents comme il en est de l'adolescente du roman de Beyala ${ }^{6}$.

3 Notamment dans Les héritiers. Les étudiants et la culture, Paris, Ed. Minuit, 1966.

4 In Education et sociologie, Paris, PUF, 1992, Rééd.; L’éducation morale, Paris, OUF, 1992, Rééd.

5 In Surveiller et punir, Paris, Gallimard, Coll. «'Tel », 1975.

6 D'ailleurs la fiction rejoint parfois la réalité. Le 31 janvier 2008, des médias français (TF1, LCI entre autres) ont diffusé en boucle l'information relative à un professeur d'un collège de Berlaimont, dans le Nord de la France, qui a fait l'objet d'une garde à vue et qui devra répondre de violence aggravée sur mineur devant le tribunal correctionnel pour avoir giflé son élève de 11 ans qui l'avait traité de "connard". On est bien loin alors de ces considérations de sociologues qui veulent que la socialisation des enfants se fasse par le biais des contraintes, de la punition, des châtiments corporels ou psychologiques (cf. Bourdieu et Passeron, Les héritiers. Les étudiants et la culture, op. cit.). 


\section{LibertÉ ET ÉDUCATION : L'ALLIANCE MALHEUREUSE}

Il serait présomptueux de donner une acception univoque de la notion de liberté tellement elle nourrit les débats. Si l'on avance ici comme opérante la définition qui entend que "l'inconditionnalité est le signe de la liberté " (Marcel, 1927 : 42), elle ne peut que poser problème lorsqu'on l'associe à la notion d'éducation et de surcroît celle des enfants qui requiert, d'une part, de l'autorité de ceux qui en ont la charge et, d'autre part, la soumission de ceux qui en sont les objets. S'il est convenu que l'une des notions fondamentales de l'éducation est de former les enfants à devenir des citoyens accomplis, c'est-à-dire des sujets libres qui jouissent d'une liberté de penser, de faire, d'agir, ou de faire un bon usage de leur liberté, il demeure que cette liberté est, si nous pouvons nous permettre l'expression, formatée car régie par les parents censés être les maîtres d'œuvre de la formation de l'enfant. À ce stade, la question que l'on est en droit de se poser est celle de savoir si les notions de liberté et d'autorité ne sont pas antinomiques si l'on admet que le principe primordial de l'éducation de l'enfant en appelle incontestablement à des restrictions donc, à une certaine limitation de sa liberté ${ }^{7}$ ?

L'exemple de Lou, la fille de l'héroïne, nous donne la preuve que l'alliance des deux notions conduit à une sorte d'oxymorisation. En effet, si Lou s'octroie tous les droits, les savoure et les outrepasse même, les devoirs, en revanche, elle estime ne pas en avoir; c'est sa mère qui en a. Il y a là un écart notable d'avec l'éducation que la narratrice elle-même aurait reçue. C'est, du moins, ce qu'elle laisse entendre lorsque s'interrogeant sur l'enfant qu'elle a été après une frasque de son " adolescente ingouvernable " (Beyala, 2007 : 90), elle a une révélation :

"C'est impossible que ma mère ait connu ça. D'aussi loin que je me souvienne, je n'ai jamais été a désobéissante, aussi insoumise, aussi cabocharde " (Beyala, 2007 : 90).

L'itération insistante de l'adverbe " aussi » pose qu'Andela au même âge que sa fille aurait été une gamine indisciplinée, mais dans une dimension bien moins importante que la diablesse dénommée Lou; le présupposé le

7 Il est de nombreux cas d'enfants qui se retrouvent à la rue ou dans des foyers sociaux pour avoir refusé de se plier aux règles de la maison, donc de leurs parents. La liberté, la "vraie ", l'enfant ne l'acquiert que lorsqu'il gagne son indépendance financière et ne risque plus de se faire couper les vivres. 
dit. Peut-être est-ce dû au milieu (l'Afrique) et aux temps (admettant que le roman de Beyala est une autofiction, l'hérö̈ne serait née en 1961; il y aurait donc trente-trois ans qu' elle a eu le même âge que sa fille, c'est-à-dire treize ans) dans lesquels elle a évolué alors. De ce point de vue, les choses ont changé dans une certaine mesure, depuis lors, surtout parmi ceux qu'il convient d'appeler les nouveaux riches qui, par une sorte de suivisme, calquent servilement le mode de vie occidental.

Sinon, dans l'éducation à l'africaine, à la différence de l'européenne, les enfants ont souvent davantage de devoirs que de droits. Toutefois, nous convenons que toutes les civilisations mettent en premier l'accent sur les devoirs en matière d'éducation car c'est à travers eux que les normes sociales sont intériorisées et que l'enfant se socialise en acquérant ce " quelque chose de puissamment générateur " que Bourdieu a dénommé "l'habitus ${ }^{8}$. En Afrique, le parent est sacré, l'enfant lui doit respect et obéissance totale. Le parent est un modèle, c'est la figure de l'idéal. La perspective de la malédiction suspendue sur sa tête comme l'épée de Damoclès est un instrument de chantage aux mains des parents lorsque leur autorité en vient à souffrir, telle l'arme de la fugue que brandissent les enfants en Europe à leurs parents pour les faire plier. Lou en use d'ailleurs, pour répondre aux réprimandes de sa mère, dans un propos comminatoire : " D'ailleurs, si tu continues à me harceler de la sorte, je fais une fugue » (Beyala, 2007 : 52). Ce à quoi sa mère répond également par la commination dans un ton ferme : «Pas de discussion. Dehors!» (Beyala, $2007: I b$.). Autant les parents, qui s'octroient d'office une autorité de droit que leur conférerait leur statut de géniteur, sont omnipotents dans la tradition africaine (même si ces temps derniers, on observe un certain relâchement ${ }^{9}$ ) autant l'autorité parentale en Europe est encadrée souvent par la loi qui s'exerce davantage que dans l'espace africain pour protéger les droits de l'enfant ${ }^{10}$. Le parent, en Europe, doit veiller à ne pas enfreindre

8 In Questions de sociologie, Paris, Ed. Minuit, 1980, p. 134.

9 On ne peut manquer ici de signaler, toutefois, la dégradation de la famille, et même sa démission de son rôle premier, souligné par certains écrivains dans des récits qui mettent en lumière l'autonmisation des enfants notamment dans des situations de conflit; c'est le cas par exemple dans Allah n'est pas obligé d'Ahmadou Kourouma (Paris, Seuil, 2000), Charly en guerre de Couao-Zotti (Paris, Ed. Dapper, 2001), Johnny chien méchant d'Emmanuel Dongala (Paris, Le serpent à plumes, 2002), pour ne citer que ceux là.

10 En réalité, il existe des principes normatifs qui devraient veiller aux droits des enfants et les protéger. Au Cameroun, par exemple, le code de la famille, la 
les lois car les enfants y veillent, les voisins également; les enseignants, les assistants sociaux sont les yeux et les oreilles des forces de police et des juges. Tout cela conduit à des situations où les parents sont lestés de leurs droits de parent, donc d'éducateur, au profit de l'enfant qui, hyper protégé, en use et en abuse à loisir. Andela semble avoir jeté les armes; ce qui, somme toute, pourrait se justifier logiquement. Comment pourrait-elle, réellement, prendre le contrôle de la situation alors que l'État le lui dénie? Que peut-elle devant une gamine qui n'obéit qu’à un principe, la liberté?

Dès la première apparition de Lou, le ton est annoncé. Au sortir d'un voyage, la narratrice appréhende déjà les "surprises désagréables [qui l'attendent] car [sa fille] Lou était une drôle de diablesse dont les macaqueries pouvaient [la] faire hurler de douleur ou pétiller de bonheur » (Beyala, 2007 : 49). Elle est dans un univers qui, au nom du respect de la liberté, droit fondamental de l'individu, dans ses postulats permet presque tout. On est tout proche de la permissivité alors qu'une des acceptions de la liberté défendue par son ardent militant, Montesquieu dans L'Esprit des lois, veut qu'elle soit le droit de faire ce que les lois admettent. Lou semble en être pleinement consciente; aussi s'autorise-t-elle tout ce que la loi ne prescrit pas mais ce qu'elle ne proscrit pas non plus. Elle passe les limites et n'a d'égard pour rien ni personne. Rosa, la dame d'un âge mur qui s'occupe d'elle, n'est rien d'autre qu'une espèce de souffre-douleur qu' elle a mis « par terre ", suivant les dires dudit personnage (Beyala, 2007 : 50). Il faut entendre par le camerounisme "être par terre ", l'expression de l'exaspération de la gouvernante que l'équivalent " être agacé », dans le français académique, n'est pas à même de rendre, dans toute sa mesure, le sentiment du personnage. D’ailleurs elle « est par terre » (Beyala, 2007: 87) au sens littéral comme au sens figuré car, c'est " assise dans les escaliers, une main soutenant ses joues comme au deuil d'une mère » (Beyala, $2007: 16$.), totalement défaite, qu'elle attend Andela pour la supplier de mettre un terme à son supplice. Aussi, on ne peut pas être surpris si les dénominatifs que Rosa associe à l'adolescente témoignent de l'aversion et de la terreur qu'elle lui inspire désormais. Il n'y est nullement question

Charte des Nations Unies sur la protection des droits de l'enfant, la Charte africaine des droits de l'homme, ainsi que le Ministère des Affaires Sociales sont sensés tous garantir juridiquement les droits de l'homme et, surtout, de l'enfant. Seulement, comme dans bien des domaines, ces derniers sont bafoués aussi bien par les parents que par la communauté dont l'enfant est membre. Il n'y a qu'à citer au nombre de ces violations le travail des enfants, l'esclavage et le trafic des enfants qui ont cours dans de nombreux pays africains. 
d'hyperbolisation car les actes et attitudes de Lou sont des preuves à charge irréfutables. Ainsi pour Rosa, c'est un monstre, expression qu'elle reprend par deux fois sous le mode exclamatif accompagné d'un juron : «Ta fille... Oh, seigneur! Quel monstre, quel monstre!» (Beyala, 2007 : 50). C'est un spécimen nouveau car, de sa longue expérience de mère, de baby-sitter et d'éducatrice, Rosa peut témoigner d'autorité et avec insistance qu'elle n'a « jamais » rien vu de la sorte :

"J'ai accouché, gardé des enfants des autres, vu en grandir d'autres, mais une comme ta fille, du jamais-vu, tu m'entends? Du-jamais-vu! » (Beyala, 2007 : 87).

On voit dans la gradation ascendante, le camerounisme « du-jamais vu ", et les modalités exclamative et interrogative qui accompagnent les propos de Rosa, la bizarrerie que représente Lou. C'est aussi une pétition d'incompétence que la gouvernante décline, par la même occasion; elle n'en peut plus de cette enfant indomptable et implore même Andela de ne plus la laisser seule avec elle.

Les enseignants font aussi les frais des libertés que s'octroie Lou. Elle joue à la spirituelle et s'amuse à tourner en dérision ses professeurs? Cela n'est guère étonnant puisqu'elle est convaincue de ce que les études ne servent à rien. Elle le traduit dans une interrogation directe à l'adresse de sa mère scandée dans un rythme triadique décroissant en cinq, quatre et trois temps qui n'est pas sans effet recherché : « Réussir sa vie, c’est étudier, selon toi? » (Beyala, 2007 : 51). L'implicite est lourd de sens puisque sa mère a abandonné le système scolaire prématurément et s'est formée au métier d'écrivaine toute seule. Lou use là d'une variante de l'argument ad personam, en l'occurrence l'argument de l'épouvantail car elle veut, sans nul doute, tourner en ridicule sa mère. Et sans lui laisser le temps de réagir, elle lui assène une seconde question tout aussi impertinente que la précédente. Dans tous les cas, elle n'attend pas de réponse car ses questions sont oratoires même si l'interpellation est directe du fait des termes d'adresse : "C'est grâce aux livres qu'on devient respectable, d'après toi? " (Beyala, $2007: I b$.). Ici encore, l'implicite est éloquent. On peut y lire le doute que la fille laisse planer quant à la respectabilité de sa propre mère qui gagne sa vie et la nourrit en écrivant des livres. Et la réponse que Lou se donne la peine d'apporter à ses propres questions n'en attend pas d'autre car elle prend la précaution d'user de la prolepse à laquelle se greffe l'apodioxis : "T'as vraiment des valeurs à chier" (Beyala, $2007:$ Id). L'univers de la gamine, auquel sa mère n'a pas accès, est un monde souffrant d'une anémie 
des valeurs et dont elle ne lui livre pas, et ne veut pas lui livrer, les clés. La seule concession qu'elle lui offre c'est de subir ses valeurs et ses humeurs.

\section{LA CONTRE-CULTURE : L'ÉDUCATION DE L'AVENIR?}

La contre-culture est un mouvement né à la faveur du surgissement du courant postmoderne dans la seconde moitié $\mathrm{du} \mathrm{xx}^{\mathrm{e}}$ siècle dans la civilisation nord américaine. Cette tendance, qui affecte en premier la jeunesse, a traversé les frontières et a gagné peu à peu le monde, à des degrés divers, instaurant des modes d'être et d'agir de groupes particuliers que l'on pourrait, à certains égards, considérer comme des marginaux, du seul fait que leurs valeurs ne sont propres qu'aux membres dudit ensemble. Parmi les caractères attachés aux sociétaires jeunes de la contre-culture figure en bonne place l'esprit contestataire en matière culturelle. Pierre Bourdieu en a une appréhension singulière :

"La désillusion collective qui résulte du décalage structural entre les aspirations et les chances, entre l'identité sociale que le système d'enseignement semble promettre ou celle qu'il propose à titre provisoire et l'identité sociale qu'offre réellement, au sortir de l'école, le marché du travail, est au principe de la désaffection à l'égard du travail et des manifestations du refus de la finitude sociale, qui est à la racine de toutes les fuites et de tous les refus constitutifs de la "contre-culture " adolescente $^{11}$ " (Bourdieu, $1979: 161$ ).

L'exemple fourni par L'homme qui m'offrait le ciel est une version édulcorée du phénomène. Exception faite de la mode hippie représentée dans le roman par la bande d'amis de la fille de la narratrice (des jeunes arborant des cheveux rouges, des ongles jaunes et des piercings à faire peur, buvant de l'alcool et fumant du hasch, Beyala, 2007 : 88), on n'a que des ramifications du phénomène. Les impératifs socio-culturels, notamment les valeurs qui fondent toute société humaine, toute civilisation, tel le respect et de surcroît celui des parents, n'existent pas dans le monde de Lou. Elle n'a qu'un seul souci, c'est être libre, libre de toute entrave. La liberté dans son appréhension c'est la liberté de faire ce qu'elle veut et, particulièrement, ce qui lui est interdit, ce qui n'est pas de son âge. C'est d'ailleurs pourquoi elle se plaît à espérer que sa mère refasse, au plus vite,

11 Pierre Bourdieu, 1979, La distinction. Critique du jugement, Paris, Ed. Minuit, p. 161. 
sa vie. Elle est persuadée de ce que "si [Andela avait] un amant [elle lui foutrait] la paix " (Beyala, 2007 : 108) comme la mère de sa meilleure amie Pauline qui laisse aller et venir sa fille à loisir. Mais, malheureusement pour elle, il n'en sera rien car la venue de François, une star de la télévision dans la vie de sa mère et, par delà elle, dans la sienne, ne va pas arranger les choses, bien au contraire. La paix à laquelle Lou aspire, en réalité, c'est celle de la déraison mais qui est plaisir dans sa conception des choses, à savoir vivre suivant le style hippie, traîner avec d'autres jeunes peu recommandables, si l'on en croit sa mère. C'est le cas de Pauline, une adolescente malicieuse, qu'Andela soupçonne fortement " d'avoir connu une cavalcade d'amants fous, que nombre d'adolescents du neuf trois avaient eu à humer sa magnifique rose noire » (Beyala, $2007: 105)$ et que Lou pose en modèle parce qu'elle, elle a le droit de faire tout ce qu'elle veut, quand elle le veut et comme elle le veut. En somme, il s'agit de développer un mode de vivre contigu de l'asociabilité. Et tous ceux qui n'y comprennent rien sont considérés comme des marginaux car, du point de vue de Lou et des siens (ses copains de bande), les valeurs des autres notamment de leurs parents, sont surannées, dépassées. Il y a là une sorte d'inversement de la pyramide des valeurs. Andela peut en témoigner car elle, plus que quiconque, est la première victime des conséquences de la rébellion de sa fille :

"Lou a une vision apocalyptique de l'univers des adultes. Pour elle, je suis une ringarde. Une caporale coincée du cerveau et des fesses. Une adjudante à la voie de chanteuse de blues qui empoisonne son existence en lui faisant subir le service scolaire obligatoire... Quant à François, puisqu'il n'est ni rappeur ni footballeur, il ne peut être à ses yeux qu'un looser. Il est trop bien habillé pour qu'elle le respecte; il est trop poli pour qu'elle l'estime. Même s'il lui prenait de jouer les Kommandatur, cela aurait sur ma fille autant d'effet qu'un pet de cafard »(Beyala, 2007 : 75)

Les valeurs de Lou s'inscrivent dans le courant contre-culturel qui se veut anticonformisme, refus, contestation de l'ordre et de l'ordinaire. Ainsi Lou instaure de nouveaux codes de fonctionnement dans la maison familiale. C'est le « je m'en foutisme " pour emprunter une expression qu'elle use à loisir dans le roman. Elle prend du plaisir à causer du déplaisir chez les personnes qui l'entourent et qui ne souscrivent pas à sa morale. L'irrespectueuse, le "monstre ", "la cancresse impitoyable " (Beyala, 2007 : 50), la " drôle de diablesse " (Beyala, 2007 : 49), c'est elle. Et elle assume ce statut avec arrogance. Le savoir vivre lui est étranger. Avec ce 
personnage on n'est pas loin du cliché habituellement brandi par certains politiques français lorsqu'il est question des banlieues françaises, notamment parisiennes. Et Lou en est une dans l'espace du roman, en premier lieu parce qu'elle y vit. Et lorsque l'on s'arrête sur ses façons d'être et surtout de se comporter, elle est une figuration du prototype du banlieusard, comme il en est des jeunes avec qui elle fait bande qui représentent une espèce de génération désenchantée qui manque de confiance dans son devenir dans la société. C'est dans une conglobation par l'accumulation des verbes d'action (six au total dans une seule phrase), figurant un monde marginal habité par la violence au quotidien, mobilisés au mode descriptif qui participe alors à présentifier l'action que la narratrice le schématise.

"Elle, [Lou] trimballait sa carcasse filiforme en compagnie des jeunes qui se battaient, roulaient par terre, se cabossaient la figure, se cassaient les dents, se frappaient et se refrappaient en s'expédiant des insultes à vous faire éclater les vertèbres : Ta mère! Le cul de ta mère! Bâtard de ta mère! » (Beyala, 2007 : 50).

On le voit, la mère, archétype sacré dans toutes les civilisations, et même mythique pour certaines de par son rôle de parturiente, est niée et ravalée au scatologique, voire au néant. Les manières d'agir de ces jeunes les rangent parmi les enfants que l'on tient généralement pour des individus sans éducation et il n'est pas jusqu'au plus petit geste qui n'en atteste pas. Les références qui commandent leur univers ne sont pas du monde du commun. Et les qualités qui leur permettent de s'affirmer se résument en des attitudes singulières qui les déclassent, les marginalisent. Lou, par exemple, s'amuse à coller des chewing-gums sur les meubles ou à se balancer négligemment dessus au risque de les abîmer. Elle est incapable, ou alors s'y refuse-t-elle, de ranger ses propres effets (vêtements, baskets, sac d'école) dont elle se débarrasse, au gré de ses humeurs, dans le premier endroit venu; et c'est le salon qui lui sert, couramment, de débarras. Les règles de bienséance lui sont inconnues, elle parle en minaudant (Beyala, 2007 : 73), ou en mâchonnant des pop-corn (Beyala, 2007 : 120), ou avec la bouche pleine (Beyala, 2007 : 124). En découvrant de telles attitudes, au fil de la lecture, on en arrive à s'imaginer que la narratrice se livre à de la caricature.

Malheureusement il n'en est rien car elle ne reproduit que, même si c'est par le biais de la fiction, la réalité sociale, celle de la jeunesse des banlieues françaises. Pierre Bourdieu, dans $L a$ distinction, a montré 
comment la socialisation concourt à l'acquisition de l'habitus et comment la hiérarchisation sociale intervient, de même, dans les manières d'être, de sentir, de percevoir des individus. Il a, par ailleurs, montré que le système scolaire participait à hiérarchiser les hommes du point de vue social, ce que l'on retrouve dans une affirmation de la narratrice qui présente les enseignants de banlieues, comme des complices du système qui s'adaptent aux habitus de leurs apprenants et ne font rien pour les en faire sortir ${ }^{12}$. Ces professeurs « savent qu'ils sont là pour préparer une fraction de leurs élèves aux grandes écoles " (Beyala, 2007 : 53); et cette minorité n'est certainement pas celle constituée par Lou et sa bande que la narratrice qualifie de "voyous » (Beyala, 2007 : 53). La voyoucratie, concept actualisé dans la France dans ce début du XXI ${ }^{\mathrm{e}}$ siècle pour désigner la délinquance juvénile et, particulièrement, les exactions des jeunes de banlieues qui entendent par ces comportements traduire leur désenchantement et plus encore un malaise social, trouve sa matérialisation dans le texte de Beyala.

Un des éléments par lesquels ces voyous se singularisent est la langue, une langue de voyous, ce que l'on a dénommé dans le monde littéraire français, au début du $\mathrm{xx}^{\mathrm{e}}$ siècle, la langue des " apaches " et dont l'empreinte était alors une espèce d'oralisation observée au niveau de l'écriture romanesque dans les structures dialogiques. La stratégie discursive de ces jeunes participe indubitablement d'une volonté d'individuation. Et même si on peut penser, à raison d'ailleurs, que la langue prêtée à Lou par la narratrice obéit à un souci de vraisemblance, le personnage campant une jeune enfant en pleine crise d'adolescence vivant en banlieue, l'on ne peut ignorer que la langue participe pleinement de l'expression d'une appréhension du monde. Aussi, cette langue plutôt familière et, parfois, prosaïque dont use Lou pour dire ses opinions et poser son être au monde, ne peut être un procédé gratuit. En effet, lorsque les énoncés produits par Lou mobilisent des vocables relevant du registre courant, le ton dans lequel elles les prononcent les ramène ipso facto dans

12 Les banlieues françaises sont tenues pour des " no man's land " ce que Sarkozy, alors ministre de l'Intérieur en France, a eu à dénommer des « zones de non droit ». L'État y a quasiment démissionné abandonnant les parias qui les peuplent à eux-mêmes, tout en laissant y proliférer une économie souterraine et un banditisme qu'il s'arrange, tout de même, à confiner dans ces zones. Lorsque ont été créées ce que l'État français a qualifié de « zones d'Education prioritaire ", il était question de prendre en charge ces jeunes de banlieues. Mais les observateurs sociaux ont montré que cela procédait plutôt d'une accentuation de la marginalisation. 
le registre familier. Son parler porte incontestablement les empreintes de son milieu. Son lexique est constitué davantage d'injures (comme en témoigne sa mère), d'apocopes, de mots élidés et grossiers. Et il faut y ajouter les tons et la kinésique qui accompagnent les propos de la jeune fille et qui, de coutume, marquent son impertinence, son mépris, son agressivité (Beyala, 2007 : 75, 105), ou son indifférence à l'égard de ses interlocuteurs. Sa mère en est souvent la première et principale victime. Et c'est généralement par le biais de la parole que la jeune fille arrive à prendre l'ascendant sur sa mère et à inverser l'ordre des choses; elle campant dans le rôle de l'adulte, du responsable, alors que la mère est reléguée, malgré elle et à son grand désespoir, au rang d'une protégée.

\section{La mère dévorée et la fille dévorante}

Au sortir de la lecture du roman de Beyala, on peut sans mal dire, parodiant la célèbre formule sartrienne, que l'enfer des parents c'est l'adolescence de leurs enfants. Les rapports entre Andela et sa fille se déclinent, d'abord, sous le sceau de la force, des violences verbale et psychologique. Il y a une sorte de renversement dialectique dans le rapport entre la mère et la fille puisque le vainqueur de l'échange n'est pas souvent la mère, mais la fille qui s'érige, au bout du compte, en censeur éthique. Mais, si ce qui prédomine incontestablement dans cette relation c'est le conflit, à des moments la mère et la fille apparaissent même comme des rivales, elles éprouvent néanmoins une affection profonde l'une pour l'autre.

\section{LA MÈRE DÉVORÉE}

Devant sa fille qu'elle qualifie, elle-même, de " carnassière " (Beyala, 2007 : 155) Andela a du mal à tenir son rôle de parent et de garante de l'éducation. Son autorité est perpétuellement contestée et remise en question par sa gamine. Toutefois, elle se refuse à abandonner Lou à ellemême alors qu'elle fait tout pour l'inciter à lui ôter toute chance de faire quelque chose de sa vie. Malgré toutes les difficultés qu'elle rencontre et qu'elle doit à sa fille, elle veut assumer son rôle, c'est-à-dire "sauver [sa fille] malgré [elle] » (Beyala, 2007 : Beyala, 2007 : 176). C'est ce qu'elle lui fait entendre dans une langue familière, alors que la jeune fille ne comprend toujours pas qu'on lui impose de «suivre [une] bonne scolarité " (Beyala, 2007 : 51) : "Ca me fout de vouloir que ma fille réussisse sa vie, qu'elle devienne quelqu'un de bien » (Beyala, $2007:$ Ib.). L'éducation que 
procure l'institution scolaire est le point d'achoppement majeur entre la mère et la fille. Dès qu'il faut réviser, elle affiche un " air farouche de taureau prêt à encorner " (Beyala, 2007 :107). Puisqu'il faut sans cesse rivaliser d'à propos avec son enfant, Andela n'a pas souvent d'autre choix que de lui opposer les mêmes armes dont elle-même use et de "réagir au coup par coup " (Beyala, 2007 : 91). C'est le cas de la langue familière, voire vulgaire à certains égards, qu'elle mobilise à dessein car c'est le seul code que Lou entende et comprenne. On en a une illustration lorsque, excédée par le comportement de son indisciplinée enfant, elle use des violences verbale et psychologique pour pouvoir s'imposer, tenir enfin sa place. Au risque de provoquer une espèce d'apathie chez Lou, Andela exaspérée n'a pas d'autre choix que de lui montrer qu'elle n'est qu'une "sale gamine " (Beyala, $2007: 88$ ) qui ne vaut rien en réalité, rien ni à ses yeux à elle sa mère à qui elle doit pourtant la vie, ni aux yeux de tous les autres censés participer, d'une certaine manière, à son éducation, à sa formation, à son devenir dans le monde. Lou est réduite par le procédé métonymique à une " gueule ${ }^{13}$ ", car sa bouche n'est propre qu'à déverser des insanités tant elle tient des propos blessants et même mortifiants. C'est principalement par cette " gueule ", ne l'oublions pas, qu'elle arrive à prendre le dessus et sur sa mère et sur les autres. Et pour lui signifier sa colère, la narratrice, dans un discours épidictique, en l'occurrence le blâme, se sert de la conglobation. On peut y retrouver des durcisseurs qui permettent à la mère de camper dans son rôle de mère :

"I'en ai marre qu'une gamine me soumette à ses desiderata. Qui es-tu pour savoir ce qui est bien pour toi ou pas? ¿e respirais l'air de ce monde

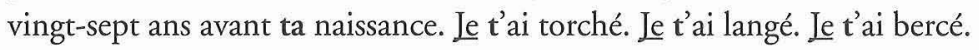
Tu n'as jamais eu faim et tu n'as jamais rien porté de plus lourd que tes

13 Il convient de relever tout le champ sémaphorique auquel renvoie le substantif " gueule » dans ce cadre. La gueule rappelle en premier la bouche des animaux auxquels elle est consubstantielle. Elle prend une coloration péjorative lorsqu'elle est associée à l'humain; mais il lui est aussi attaché toute une symbolique menaçante; les auteurs du Dictionnaire des symboles, Jean Chevalier et Alain Gheerbrandt, la tiennent pour un "symbole chtonien - ou infernal - », Paris, Laffont, 1986, p. 491. Pour Gilbert Durand, c'est dans « la gueule animale que viennent se concentrer tous les fantasmes terrifiants de l'animalité : l'agitation, manducation agressive, grognements et rugissements sinistres ", in Les structures anthropologiques de limaginaire, Paris, Dunod, 1991, $11^{e}$ éd., p. 74. 
jeans et tes baskets que je me gaspille les yeux devant un ordinateur pour pouvoir t'acheter. Qui es-tu pour désapprouver tes professeurs ou te moquer d'eux? Qui es-tu pour répondre aux gens avec mépris? Tu sais en réalité ce qu'ils pensent tes profs? Qu'ils n'ont rien à foutre de ta gueule! Ils savent qu'ils sont là pour préparer une fraction de leurs élèves aux grandes écoles. Quant aux merdes comme toi, parce que vous n'êtes que cela, toi et tes petits voyous d'amis, ils se disent que s'ils réussissent à vous amener à résoudre un problème d'arithmétique du cours élémentaire, ils auront réussi leur mission, tu piges? Maintenant dehors!" (Beyala, 2007 : 52-53).

Elle y mêle des actes illocutoires interrogatifs, en réalité ce sont des questions oratoires (cinq au total) pour l'intimer au silence; des arguments de l'épouvantail pour la ridiculiser; d'autorité par l'expérience et ad verecundiam (ses statuts de génitrice et d'éducatrice les lui octroient d'office), d'autorité de droit (les sacrifices auxquels elle s'oblige depuis la naissance de Lou en sont la preuve), de la compétence (les professeurs en sont pourvus); de communauté (les valeurs de respect sont communes à toutes les civilisations). Le lexique est constitué de mots de registre familier (en avoir marre, torcher, foutre, gueule, merde, piger, l'apocope " profs"). L'instrument rhétorique qu'elle convoque participe du ton sermonnant de la narratrice comme on peut le lire dans la gradation ascendante triptyque accompagnée d'une reprise anaphorique : «Je t’ai torchée. Je t'ai langée. Je t'ai bercée. ". Il est manifestement question pour Andela de faire entendre, de faire savoir et de faire comprendre, de faire prendre la mesure de son sacrifice à sa fille. La structure phrastique, en majorité constituée de phrases simples et courtes, donne ainsi au style de la narratrice un ton incisif, lapidaire, pour marquer aussi l'esprit de l'adolescente. L'analyse du système énonciatif fait apparaître, en quatorze phrases, vingt-six appellatifs dont vingt-deux pronoms personnels et adjectifs possessifs désignatifs de l'interlocuteur, donc de Lou, et seulement six référents du locuteur, la mère. Les termes d'adresse par leur forte présence prennent ici la valeur de durcisseurs car il n'est nullement question pour Andela d'épargner sa fille. Il faut croire qu'elle voulait frapper fort car au terme de sa diatribe, Andela est fière d'elle; elle avoue même qu'elle « [venait] de gagner une belle partie de poker» (Beyala, $2007: 53$ ).

Hannah Arendt affirme, dans La crise de la culture, que « l'autorité a été abolie par les adultes " (Arendt, 1972 : 234). Et elle n’avait pas tort car en se félicitant d'avoir gagné la partie dans cette sorte de combat qui l'a opposé à sa fille (toute la scène figure deux boxeuses se défiant sur un ring), 
ce qui ne devrait pas être, Andela reconnaît, non seulement, son incapacité à pouvoir l'éduquer, mais aussi sa démission implicite de ce devoir. L'équation de la relation filiale parent-enfant qui veut que ce soit le parent qui donne des ordres et l'enfant qui obéisse, est totalement inopérante dans L'homme qui m'offrait le ciel. La narratrice s'essaye, comme dans une banale partie de cartes, à son rôle de parent, d'éducatrice. En réalité, elle n'a pas d'autre choix car, l'histoire montre bien que c'est Lou qui mène son monde par le bout du nez. Sa mère arrive à rebondir de rares fois comme il en est dans l'exemple évoqué ci-dessus. Autrement, c'est Lou qui, en véritable manipulatrice, provoque sa mère, lui fait perdre pied, de sorte que cette dernière en est arrivée à la redouter. La maison familiale, n'est pas un havre de paix comme on aurait pu s'y attendre, mais une arène de lutte, un " terrain $[\ldots]$ piégé, si miné " que [c'est la mère qui prend] garde à où [elle met] les pieds " (Beyala, 2007 : 75). La mère se retrouve bien souvent dans la position de celle qui doit parer les coups; et rappeler aussi à sa fille que c'est elle l'adulte qui pose les règles mais, il faut le dire, sans grand succès. Aussi n'est-t-on pas surpris lorsqu' elle confesse que son " destin de mère [l'] accable (Beyala, 2007 : 91).

Il faut dire que "sa majesté la cancresse " (Beyala, 2007 : 106) Lou, ne lui laisse aucun répit. Toutes les apparitions de l'adolescente, il y en a sept au total dans le roman, sont des moments névralgiques car ils figurent toujours des antagonismes mettant au jour, au premier chef, le conflit entre la mère et la fille qui devient, en plus, le lieu pour poser des questions d'utilité sociale. La tension va redoubler lorsque François va s'immiscer dans ce duo; Andela sera alors davantage malmenée par sa gamine. Et c'est d'autant plus irritant pour la narratrice que les réflexions impertinentes de sa fille sont, bon gré mal gré, idoines. Et les réactions d'Andela, à chaque fois que sa fille argumente sur sa relation avec sa star de télévision, qui parlent d'elles-mêmes, en attestent. Généralement, elle, l'adulte, agacée et complètement désarmée, perd son calme et finit par agresser :

"Exaspérée, je donnai un coup de poing sur la table:

- Ma vie privée ne te regarde pas, OK?

La théière se renversa et se brisa en mille morceaux que je me mis à ramasser méthodiquement un par un, pour ne pas méditer ce que ma fille proférait "(Beyala, $2007: 156)$

Cette gamine impudique mais étonnamment lucide, s'agissant des amours de sa mère, essaie tant bien que mal de faire recouvrer la vue à une femme aveuglée par un amour manifestement impossible et qui s'obstine à 
nier les évidences. Aussi, lorsqu'à la fin, à bout de force et de nerfs après une énième indélicatesse de sa fille elle se décide à l'envoyer en pension, on a comme l'impression que ce n'est pas pour la raison invoquée, à savoir sauver cette enfant malgré elle. Même s'il est indéniable que cette institution disciplinaire doit jouer un rôle de socialisation de cette gamine marginale en lui réapprenant les valeurs fondamentales de la société, on peut aussi croire que c'est pour se sauver elle-même de cette enfant turbulente que la narratrice se résout à s'en séparer. Elle lui cause de plus en plus d'angoisses au point qu'elle en est arrivée à assimiler son destin de mère à un état proche de la damnation. Lou est, dans ses conditions, comme une manifestation de l'horreur, une sorte de vermine qui grignote sa mère à petits coups térébrants.

\section{LA FILLE DÉVORANTE}

De toutes les analyses bâties jusqu'à ce stade, il apparaît que Lou figure une sorte d'ogresse. C'est une manipulatrice qui se plaît à conduire le monde qui l'environne, en particulier sa mère, selon ses humeurs. Elle a investi l'espace que représente la maison familiale et y affiche des airs de

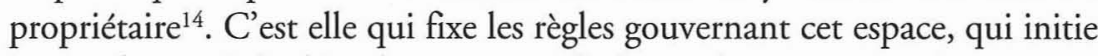
les combats qui s'y déroulent et en est l'arbitre, du moins jusqu'à ce que sa mère, concédant son impuissance, trouve l'arme imparable : la pension. C'est cette institution qui devra se charger de redresser l'adolescente qui suggère que sa mère et son amant entendent, par ce moyen, se débarrasser d'elle pour couler des jours paisibles sans avoir à s'inquiéter de ses frasques et à essuyer son venin.

Mais jusque-là, l'adolescente agit au gré de ses caprices, s'en amusant parfois. Seuls ses désirs comptent alors et prévalent sur ceux des autres, surtout ceux de sa mère. On peut alors penser qu'elle en a après elle spécialement. On peut le lire dans cette invective, la première du reste, dans le roman, qu'elle formule à l'encontre d'Andela alors que celle-ci essaie de la raisonner après un écart de conduite : « Je m’en fiche. Je ne t’ai

14 Nous l'avons évoqué en analysant les attitudes de Lou dans la maison familiale. Sa mère va essayer de la contraindre à tenir sa place en la contraignant à s'occuper de ses effets personnels, notamment ses vêtements qu'elle a pris l'habitude de jeter négligemment dans le salon sans y parvenir avec succès. L'avant dernière scène dans laquelle l'adolescente apparaît dans le roman la représente de retour de classe; et son premier geste, comme à l'accoutumée, est de se débarrasser de son blouson sur le canapé dans le salon avant de commencer d'invectiver sa mère (Beyala, 2007 : 154). 
rien demandé, moi. »(Beyala, 2007 : 51). L'implicite laisse entendre nombre de choses. Cependant, l'élément significatif central qui découle des propos de Lou, c'est qu'elle n'a pas demandé à naître. Et du seul fait d'avoir pris la responsabilité de lui donner la vie, sa mère s'est engagée également à assumer ce qui va avec; justement ce qu'elle présente comme des sacrifices aux yeux de sa fille mais qui, en réalité, constituent sa charge de parent. C'est son devoir de parent de s'acquitter des frais de la "bouffe", des «fringues ", du coiffeur, des cahiers, parce qu'elle, Lou, n'est qu'une enfant comme elle se fait fort de lui rappeler insolemment : " T'es pas sérieuse, tout de même! Je ne suis qu'une enfant » (Beyala, 2007 : 55). Mais une enfant qui ne se rend compte de ce statut que lorsque la situation l'arrange. Si elle revendique haut et fort les droits de se faire entretenir grassement par sa mère qui n'a pas d'autre alternative (si ce n'est de la refiler aux services sociaux et être taxée de mauvaise mère), elle refuse ostensiblement à cette mère, si précieuse lorsqu'il s'agit de pourvoir aux besoins matériels, d'assumer la part éthique de son éducation, lui déniant toute qualité pour s'en charger.

Lou se veut, pour l'occasion, moralisatrice. Et elle ne manque pas une opportunité de redire à sa mère ses responsabilités. Et ces dernières ne vont pas sans les qualités du parent. Le parent, est-il encore besoin de le rappeler, représente un parangon auquel l'enfant se réfere soit pour s'identifier, soit pour s'en différencier. Et si Lou n'arrive pas à s'expliquer que sa mère ne comprenne pas son désintérêt manifeste pour l'école, c'est bien parce qu'elle-même n'est pas un modèle de réussite par ce biais. Elle jette un regard sans concession sur sa mère, l'amenant à une sorte d'introspection. C'est le cas quand elle la surprend en peignoir en pleine après-midi, alors qu'elle ignore encore que sa mère entretient une relation adultérine avec François :

" Reconnais que ça fait mauvais genre une mère pas habillée à presque six heures du soir. $\mathrm{Ca}$ me rappelle les mères alcoolos et les mères putains qu'on voit dans les films. Tu ne trouves pas? » (Beyala, 2007 : 106).

Et l'on peut comprendre alors qu'elle ait traîné ostensiblement, devant la maison, avec sa copine Pauline, repoussant l'invitation de sa mère à la rejoindre, à son retour des classes. La scène se poursuit par les reniflements de la jeune fille qui, tel un chien policier à la recherche de l'indice irréfutable qui condamnerait sans conteste un prévenu, veut prendre sa mère en défaut en dénichant l'objet du délit qui l'accablerait. Ce qu'elle ignore, c'est que, bien avant qu'elle n'affiche son attitude inquisitrice, 
accusatrice et méprisante, elle avait marqué un point avec le coup de la mère prostituée. En effet, sa maman toute honteuse " [cherchait déjà] une grotte où [se] cacher » (Beyala, $2007: I b$.), confessant, du même coup, sa culpabilité. "C'est toujours en se servant d'un langage choc constitué de mots que Brice Parrain, cité par Jean-Paul Sartre $(1948,31)$ qualifie de "pistolets chargés". "

L'autre devoir de parent qui incombe à Andela c'est d'être présente pour sa fille. Ainsi on peut, logiquement, concevoir que Lou, par son impudence, veut faire payer à sa mère ses multiples absences, d'autant plus qu'elle est privée de l'affection de son père dont il n'est fait mention à aucun moment dans le roman. Et elle se fait fort de le remémorer à sa mère qui, à peine rentrée d'un voyage, en pleine année scolaire, l'abandonne aux mains d'une Rosa analphabète et passe son temps à roucouler dans son lit ou à battre les pavés des restaurants huppés avec son amant. Elle l'interpelle alors comme pour la rappeler à ses devoirs «Tu sais que l'absence des parents est la cause essentielle de l'échec scolaire des enfants? 》 (Beyala, 2007 : 74). Aussi, les conditionnalités familiales sont d'une importance déterminante dans l'analyse de la situation. La mère dans le cadre de la famille a certes de l'autorité, mais le père l'a plus encore. Or, le père de Lou est totalement absent pour ne pas dire invisible, et la mère, (la seule autorité parentale) est absentéiste, occupée qu'elle est à courir le monde pour livrer des combats ou en escapade amoureuse. Lou, on peut le dire, souffre d'une sorte de carence paternelle que sa mère ne semble pas à même, ni ne veut, combler. Andela se refuse manifestement à jouer le rôle de la « bonne mère » que le psychanalyste Michel Schneider pose comme étant "d'inculquer à l'enfant qu'il y a des limites qui passent par l'autorité paternelle ${ }^{15}$ ». Â aucun moment elle ne fait recours au père de cette enfant dont le symbole, à lui seul, est fortement puissant dans l'imaginaire des enfants. Ce père est non seulement absent physiquement, mais il est aussi marginalisé par la mère qui l'a complètement soustrait de leur univers. Or, il est le seul qui aurait pu enseigner à sa fille les codes opératoires sociaux qui lui font tant défaut et, pourquoi pas, la ramener à la raison.

Rosa, sorte de succédané de la mère, n’a aucune chance de parvenir à un résultat positif. Elle ne possède pas les armes nécessaires pour s'y adonner à dessein; d'ailleurs, la jouvencelle ne lui a pas concédé une semblable opportunité. C'est de sa mère dont elle a besoin et non d'un substitut de mère. C'est pourquoi elle fera en sorte que Rosa se souvienne

15 Michel Schneider, Big mother, psychopathologie de la vie politique, Paris, Odile Jacob, 2003. 
à chaque fois qu'elles se retrouvent, en l'absence d'Andela, qu'elle n'est pas sa mère et lui fera payer, à sa manière, de jouer ce rôle qui ne lui est pas dévolu. On a alors l'impression de faire face à une espèce de relation sadomasochiste entre l'adulte et l'enfant. Il n'est donc pas surprenant que la pauvre Rosa en arrive à implorer sa patronne de la soustraire aux "turbulences" (Beyala, 2007 : 174) de sa fille; elle se refuse ainsi à jouer le rôle de fusible. Mais, il faut aussi y voir, non seulement une invite à Andela à assumer le rôle d'éducatrice qui lui incombe au premier chef, mais aussi le reproche tacite d'avoir failli à l'éducation de son enfant.

La narratrice est, à nul doute, dépassée par les agissements et attitudes de sa jeune fille. Il faut dire que l'entrée de François dans sa vie lui fournit un alibi pour se défiler d'une tâche qu'elle ne parvient pas assumer véritablement. Mais il faut reconnaître qu'elle n'est pas la seule responsable et c'est Lou, elle-même, qui le lui concède. Alors que l'adolescente veut encore l'accabler, la réplique de la mère, qui se refuse à endosser toute seule la responsabilité de l'échec de son éducation, est incisive :

" Je ne vais pas culpabiliser, Lou. J’ai toujours été présente. Tu n'es pas pour autant un modèle de réussite " (Beyala, 2007 : 74)

L'adolescente, de son propre aveu, reconnaît qu'elle n'est pas un bon exemple ce qui, par ailleurs, conforte sa génitrice qui est ainsi absoute de ce que Lou posait déjà comme une faute grave, ses absences répétées. Toutefois, il serait logique de croire que ces absences ne sont pas sans conséquence sur le mode de vie adopté par la gamine qui fait désormais fi de la présence de sa mère lorsqu'elle a l'heur d'être présente. Andela le lui rappelle d'ailleurs à dessein alors qu'elle lui fait remarquer l'incongruité de la situation dans l'image de cette enfant, Lou, qui se réclame telle " mais qui peut sortir jusqu'à deux heures du matin sans avoir peur du grand méchant loup " (Beyala, 2007 : 55).

Même si l'on accrédite la thèse d'une probable rancune de la fille à l'égard de la mère, on doit aussi reconnaître qu'elle a une réelle affection pour sa mère ce que corrobore, d'ailleurs, l'écrivaine dans une interview. Elle affirme que, par-delà les rapports conflictuels qu'entretiennent ses deux personnages, elles entretiennent une relation " très affectueuse ${ }^{16}$ ». En effet, l'adolescente veille aux intérêts de sa mère, même si ça l'est aussi, il faut le dire, par pur intérêt égoïste, car elle veut l'avoir à elle toute seule.

16 Cf. le site Afrik.com 
Toujours est-il qu'elle fait davantage preuve de clairvoyance que sa mère, l'adulte dans sa relation avec son amant. Au premier contact avec celui qui aurait pu devenir son beau-père elle confie à sa mère ses appréhensions.

\begin{abstract}
«Ma fille me dit qu'elle n'avait pas confiance en François. Qu'il était faux comme une photocopie. Qu'elle craignait qu'il ne montrât de lui qu'une voltige de lucioles dans la nuit, des chatoiements de surface d'une rivière aux fonds boueux, des flamboiements de rosée matinale dans une forêt tropicale. Non décidément, il n'était pas trop fiable, trop lisse pour ne pas être complexe, trop généreux pour ne pas cacher d'horribles stratégies " (Beyala, 2007 : 122).
\end{abstract}

Et ses premières impressions, que l'on pourrait croire guidées par la jalousie comme sa mère en est persuadée alors, se vérifieront par la suite. François se montrera avare n'offrant aucun cadeau à sa dulcinée, comme il est de coutume dans cette société-là alors qu'il en a les moyens, égocentrique, occupé et préoccupé qu'il est de sa personne. Le mépris de Lou à son égard n'en sera alors que plus grand; elle "le regardât comme le pet d'un cafard "(Beyala, 2007 : 173). Les rôles sont inversés. C'est l'enfant qui, plus perspicace, flaire le danger et entreprend de refaire l'éducation amoureuse de la mère alors qu'elle devrait prendre des leçons auprès d'elle. Peut-être espère-t-elle ainsi limiter les dégâts. Elle n'hésite pas alors à interpeller sa mère, même si pour cela elle doit utiliser la raillerie :
«Même les adolescents ne font pas comme vous, maman [...]
Même à mon âge, on ne passe plus son temps à se dire je t'aime, à rester collés l'un à l'autre comme des sangsues. Même moi je sais que les je t’aime ne remplissent pas le caddie " (Beyala, $2007: 156$ ).

Et si les moments tendres entre Andela et Lou, dans la narration, sont peu nombreux, à la différence des disputes, ils témoignent pourtant d'un lien profond entre la mère et la fille qui se manifeste, avouons-le, de façon curieuse. Un exemple nous est fourni lorsque la narratrice entreprend de décrire un instant singulier d'après friction avec l'adolescente.

"Lou se fit une place dans la baignoire à la pousse-toi- que je m’installe. Elle aimait ça, se baigner avec moi, me montrer son corps gracile, son ventre plat, ses muscles longs, tout ce que moi-même j'étais il y a quelques années " (Beyala, $2007:$ 54). 
La scène figure deux amies se prélassant dans une baignoire, s'admirant et savourant précieusement un laps de temps. On ne peut manquer de s'interroger quant à cette proximité qui, à certains égards, peut paraître promiscue, si l'on prend en compte la relation filiale entre les deux personnages, De deux sœurs, l'appréhension en aurait été différente, puisqu'elles appartiendraient sensiblement à la même génération. Cette proximité, somme toute touchante, peut expliquer le fait que l'adolescente ne parvienne pas toujours à opérer la différence entre l'amie et la mère en Andel $\mathrm{a}^{17}$. L'altercation pourtant violente n'est plus qu'un lointain souvenir. L'affection singulière de la jeune fille pour sa mère s'affiche davantage lorsqu'elle prend sur elle de soutenir sa mère qu'elle sent fragile à cause de sa relation avec François. Elle s'en ouvre par ailleurs à cette dernière : " cet amour te fragilise tant que j'ai envie de te protéger" (Beyala, 2007 : 157). On découvre, dans la dernière partie du roman, une autre Lou plus attentionnée, plus responsable, et plus prévenante même si elle n’a pas perdu de sa rudesse. Mais sa verdeur, cette fois, qui s'est muée en " condescendance " (Beyala, 2007 : 155), est liée à sa volonté d'éveiller sa mère à ce qui se passe et dont elle est la victime. C'est, en outre, les seules fois où on la voit, dans le roman, effectuer une quelconque tâche, tels qu'offrir du thé à sa mère en s'enquérant au préalable du parfum qu'elle désire, la câliner.

À la confiance totale que sa mère affiche à l'endroit de François, elle oppose ses réticences et son mépris outrancier. Elle a peur que l'amant d'Andela ne soit " en train de se payer un caprice d'enfant gâté " (Beyala, 2007 : 122). Les tentatives répétées de François pour gagner son affection l'agacent et contribuent plutôt à accroître son insolence. Elle considère Ackerman comme un intrus qui profite de la crédulité de sa mère et entend le traiter en conséquence. Et le sexagénaire s'en est bien rendu compte et le confesse à sa dulcinée : "Elle me déteste parce qu'elle croit que je lui vole sa mère " (Beyala, 2007 : 173). Mais la réalité est toute autre. Lou se refuse à ce que sa maman se « laisse couillonner longtemps » (Beyala, 2007 : 154),

17 Cela participe, peut-être, de cette éducation nouvelle qui veut que l'on soit proche de l'enfant. Mais il faut dire que la frontière est tenue pour l'enfant qui préfere bien évidemment le parent-ami plus souple, plus malléable au parentparent (sorte de parent fouettard) qui use de son autorité. Dans l'univers africain, par exemple, il ne viendrait pas à l'esprit de l'enfant de discuter des affaires de cœur de son parent avec celui-ci et encore moins des siennes; tout ce relevant habituellement du sexe appartenant à un domaine tabouisé.48 La Vie promise, op. cit., p. 225. 
continue d'être "Pathétique et conne. Conne et idiote. Idiote " (Beyala, 2007 : 155). Au vrai, la jeune fille n'arrive pas à s'expliquer cette apathie de sa mère alors qu'elle l'a habituée et éveillée, jusque-là, au militantisme et à la hardiesse. Aussi, comment cette femme si perspicace et avisée lorsqu'il s'agit de questions plus complexes peut-elle manquer d'autant de jugement lorsqu'il s'agit de sa propre personne? Telle est la préoccupation de Lou qui est la seule, avec Rosa, à avoir flairé la déception au devant de laquelle allait Andela. C'est encore elle qui va essayer d'insinuer le doute dans l'esprit de sa mère quant à la sincérité des sentiments de son amant et la viabilité de leur histoire d'amour en posant la question cruciale de la race, sans vraiment parvenir à l'ébranler :

« Mais je m'interroge. Ca fait un an que vous êtes ensemble. Pourquoi n'officialise-t-il pas votre relation? Ce n'est tout de même pas parce que tu es noire, maman. » (Beyala, $2007:$ 157)

En fait de question, c'est plutôt une thèse que Lou avance, car l'interrogation est posée sous une forme déclarative. De fait, elle invite sa mère à envisager, avec tout le sérieux que cela mérite, le doute qu'elle a levé. Et les arguments dont elle se sert ne sont pas spécieux. Elle fait constater opportunément à la narratrice que " les invités de François sont presque tous des Blancs " (Beyala, 2007 : 158). Et comme sa mère continue d'être sourde et aveugle à ce qui se passe, elle va employer les grands moyens pour la "faire tomber dans un trou sans fond » (Beyala, 2007 : 174). Elle va lui user les nerfs en multipliant les frasques. Elle a pris à tâche de briser ce couple pour rendre à sa mère une dignité qu'elle a perdue dès le moment où elle est tombée amoureuse. Lou en sera quitte pour son éloignement du foyer familial. Sa conclusion alors est des plus éloquentes : "Si tu ne t'es pas encore rendue compte que ton François n'abandonnera jamais son confort pour l'amour, alors, t'es qu'une imbécile» (Beyala, 2007 : 176). Et imbécile la narratrice le fut, en fin de compte. Abandonnée lâchement par son amant, pleurant sur elle-même, Andela se remémora les appréhensions de sa fille qui prirent ainsi tout leur sens, dès cet instant, provoquant chez la narratrice, ce que sa fille a recherché avec acharnement, l'instinct de révolte endormi par l'amour :

« Lou avait émis l'hypothèse raciale comme frein à notre amour. Au lieu de ça, j'avais ri de bon cœur en dévorant mes escargots. J'avais ri avec insouciance en montrant mes trente-deux dents et en savourant un verre de champagne " (Beyala, 2007 : 207). 
L'adolescente n'est pas là pour voir les tourments de sa mère, ou la railler car elle l'avait prévenue, et encore moins panser ses blessures. Aurait-t-elle été là que cela aurait changé quelque chose? Rien n'est sûr.

Inconsciemment, Lou en soulevant le problème racial et en l'inscrivant comme un empêchement à la survivance de la relation de sa mère avec son amant, François, pose le problème des valeurs dans un sens plus large. Il n'est plus alors question uniquement de l'éducation ou uniquement des rapports interraciaux, mais de valeurs humaines et sociales, en général, au rang desquelles le respect de l'homme tient une place des plus importantes. Les questions que l'on doit poser dans ce cadre sont celles de savoir comment élever un enfant issu de la miscégénation dans un monde qui opère des différences entre les êtres humains et ravalent certains au rang de sous-hommes, dans un monde où le puissant sentiment qu'est l'amour ne réussit pas à transcender les barrières raciales? Comment lui inculquer des valeurs de paix, de tolérance et de respect dans un monde permanemment soumis à la violence sous diverses formes? C'est ce que semble traduire le comportement irrespectueux de Lou. L'effronterie de la jouvencelle est de l'ordre du questionnement. Ces attitudes sont autant de questions qui se posent à la société humaine dans son entièreté. Est-ce à dire que la situation est irréversible et que le monde s'achemine vers le chaos, qu'il est condamné à s'effondrer? L'homme qui m'offrait le ciel laisse des blancs mais s'achève en quelque sorte sur une note d'espoir. Lou est en pension il n'est plus question d'indiscipline. Et si l'on admet que la présence de François a contribué à décupler son impertinence, on peut aussi légitimement croire que son départ de la vie de sa mère, conséquemment de la sienne, ne peut que rapporter une sérénité relative dans les rapports entre la mère et la fille qui pourraient même retrouver une complicité. Aussi, on peut dire que tout n'est pas noir.

\section{Conclusion}

Le problème de l'éducation que pose le roman de Beyala est des plus actuels. Les temps à venir, du fait des évolutions technologiques et des appréhensions du monde qu'elles induisent, vont contribuer à affaiblir davantage l'autorité parentale. L'exemple de la relation entre Andela et sa fille Lou, bien que fictif mais pas si loin de la réalité, montre à suffisance la difficulté de plus en plus grande des parents à élever un enfant dans des temps et des espaces qui évoluent constamment et pas toujours positivement. En effet, si le parent a à cœur de participer au devenir de son enfant, ce dernier ne lui laisse pas toujours l'occasion, et encore moins les 
moyens, de lui procurer une éducation positive et idoine. L'enfant, comme il en est de Lou dans le roman, revendique de plus en plus une certaine autonomie qui n'est pas sans risque pour lui. Peut-être, aussi, que les enfants devraient prendre la mesure de la responsabilité qu'ils s'octroient et être lucides pour construire convenablement leur identité et leur être au monde. Les parents doivent-ils pour autant abdiquer, tel Andela dans le roman? Non, car il en va du devenir du monde; et seule l'éducation dont le rôle premier est d'œuvrer à l'instauration et à la promotion de vertus fondamentales tels la fraternité, le respect et la solidarité entre les hommes est à même de mener cette bataille.

\section{Bibliographie}

Arendt, H., (1972), La crise de la culture, Paris, « Folio ».

Beyala, C., (2007), L'homme qui m’offrait le ciel, Paris, Albin Michel.

Bourdieu, P., (1979), La distinction. Critique du jugement, Paris, Minuit.

Bourdieu, P., Passeron, J.-C. (1966), Les héritiers. Les étudiants et la culture, Paris, Minuit.

Foucault, M., (1975), Surveiller et punir, Paris, Gallimard, Coll. « Tel ». Marcel, G., (1927), Journal métaphysique, Paris, Gallimard.

Morier, H., (1989), Dictionnaire de poétique, Paris, Seuil.

Sartre, J. P., (1948), Qu'est ce que la littérature? Paris, Gallimard. 